\title{
EXTENSIONS OF STRICT PARTIAL ORDERS IN $N$-GROUPS
}

\author{
K. B. PRABHAKARA RAO \\ (Received 20 April; revised 5 August 1977) \\ Communicated by $\mathbf{H}$. Lausch
}

\begin{abstract}
An attempt is made to extend the theory of extensions of partial orders in groups to strict partially ordered $N$-groups. Necessary and sufficient conditions, for a strict partial order of an $N$-group to have a strict full extension, and for a strict partial order of an $N$-group to be an intersection of strict full orders, are obtained when the partially ordered near-ring $N$ and the $N$-group $G$ satisfy the condition $(-x) n=-x n$ for all elements $x$ in $G$ and positive elements $n$ in $N$.
\end{abstract}

Subject classification (Amer. Math. Soc. (MOS) 1970): 06 A 75.

\section{Introduction}

In this paper we attempt to generalize the theory of extensions of partial orders in groups (Fuchs, 1963) to strict partially ordered $N$-groups. The paper is divided into five sections. In Section 1 we present preliminaries. In Section 2 a necessary and sufficient condition for the partial order of a strict partially ordered $N$-group to have a strict full extension is obtained. In Section $3 \mathrm{~N}$-groups admitting strict full orders are discussed. Section 4 deals with $N$-groups, in which every strict partial order can be extended to a strict full order. In Section 5 necessary and sufficient conditions, for the partial order of a strict partially ordered $N$-group to be an intersection of strict full orders, are investigated.

A near-ring is a system $N=(N,+, ., 0)$ satisfying

(i) $(N,+, 0)$ is a group;

(ii) $(N$, .) is a semigroup;

(iii) $x(y+z)=x y+x z$ for all $x, y$ and $z$ in $N$;

(iv) $0 x=0$ for all $x$ in $N$.

From (iii) it can be easily deduced, that $x 0=0$ and

$$
x(-y)=-x y \text { for all } x, y \in N .
$$


Let $N$ be a near-ring. An additive group $G$ is called an $N$-group if there exists a mapping $(g, m) \rightarrow g n$ of $G \times N$ into $G$ such that

(i) $g(n+m)=g n+g m$; and

(ii) $g(n m)=(g n) m$, for all $g \in G$ and $n, m \in N$.

If $\overline{0}$ is the identity of $G$, using (i), it is easy to verify that $g 0=\overline{0}$ and $g(-n)=-g n$ for all $g \in G$ and $n \in N$. Also $\overline{0} n=(\overline{0} 0) n=\overline{0}(0 n)=\overline{0} 0=\overline{0}$.

A normal subgroup $K$ of an $N$-group $G$ is called an $N$-kernel if $(x+y) n-y n \in K$ for every $x \in K, y \in G$ and $n \in N$. The definition $(x+K) n=x n+K$ makes the quotient group $G / K$ an $N$-group.

A group $(G,+)$, together with a partial order $\leqslant$ on $G$, is called a partially ordered (po) group if $a \in G, b \in G, c \in G$ and $a \leqslant b$ imply $a+c \leqslant b+c$ and $c+a \leqslant c+b$ (Fuchs, 1963). The set of all positive elements of $G$, that is, $\{x \in G: 0 \leqslant x\}$ is called the positive cone of $G$.

Pilz (1971) defined a po near-ring, by imposing the requirements that it must be a po group under addition and that the product of two positive elements must be positive. Radhakrishna (1975), p. 24 observed that this definition does not guarantee that the product of two negative elements is positive. He defined a po near-ring, ensuring that the product of two negative elements is positive.

Definition 1.1. (Radhakrishna (1975).) A near-ring $N$ is called a partially ordered (po) near-ring if $(N,+)$ is a po group under a relation $\leqslant$, and $a \leqslant b, 0 \leqslant c$ imply $a c \leqslant b c$ and $c a \leqslant c b$.

Let $N$ be a po near-ring. The set of all positive elements of $N$, that is, $\{x \in N: 0 \leqslant x\}$ is called the positive cone of $N$ which we shall denote by $P(N)$. $P(N)^{*}$ stands for the set $P(N) \backslash\{0\}$.

Definition 1.2. Let $N$ be a po near-ring. An $N$-group $G$ is called a partially $\operatorname{ordered}(p o) N$-group if $G$ is a po group under a relation $\leqslant$, and $x, y \in G, x \leqslant y$, and $n \in \boldsymbol{P}(N)$ imply $x \boldsymbol{n} \leqslant y n$.

Now we obtain a characterization of the positive cone of a po $N$-group.

Lemma 1.3. Let $N$ be a po near-ring with positive cone $P(N)$. A subset $P$ of an $N$-group $G$ is the positive cone of some partial order of the $N$-group $G$ if and only if $P$ satisfies the following three conditions.

(i) $P$ is a normal subsemigroup of $G$ containing 0 .

(ii) $P \cap-P=\{0\}$, where $-P=\{x \in G:-x \in P\}$

(iii) $x \in P, y \in G$ and $n \in P(N)$ imply $(x+y) n-y n \in P$.

Proof. Suppose that $P$ is the positive cone of some partial order $\leqslant$ of the $N$-group $G$. Clearly, $P$ satisfies (i) and (ii) (Fuchs, 1963, p. 13). Let $x \in P, y \in G$ and $n \in P(N)$. Since $0 \leqslant x$, we have $y \leqslant x+y$ and $y n \leqslant(x+y) n$. Hence

$$
0 \leqslant(x+y) n-y n \text {. }
$$


Conversely, let us suppose that $P$ satisfies (i), (ii) and (iii). Define $x \leqslant y$ if and only if $y-x \in P$. Then $G$ is a po group under the relation $\leqslant$, and $\{x \in G: 0 \leqslant x\}=P$ (Fuchs, 1963, p. 13). Let $x, y \in G$ such that $x \leqslant y$. Let $n \in P(N)$. Now

by (iii).

$$
y n-x n=(y-x+x) n-x n \in P
$$

Definition 1.4. Let $N$ be a po near-ring with positive cone $P(N)$. A po $N$-group $G$ is said to be a strict po $N$-group if $x, y \in G, x<y$ and $n \in P(N)^{*}$ imply $x n<y n$.

EXAMPLE 1.5. Let $G$ be a po group. Let $Z$ be the ring of integers, with the usual order. For $x \in G$ and $z \in Z$ define

$$
x z=\left\{\begin{array}{l}
x+x+\ldots+x, z \text { times if } z>0, \\
(-x)+(-x)+\ldots+(-x),(-z) \text { times if } z<0, \\
0 \text { if } z=0 .
\end{array}\right.
$$

Then $G$ is a strict po $Z$-group.

The following example is due to Radhakrishna (1975), p. 23.

EXAMPLE 1.6. Let $R$ be the set of all polynomials of the form $f(x)=\sum_{i=1}^{n} a_{i} x^{i}$ with $a_{i}=0$ for even $i$, over an ordered integral domain. $R$ is a fully ordered group under the componentwise addition, and under the lexicographic ordering, that is, $0<f(x)=\sum_{i=1}^{n} a_{i} x^{i}$ if the coefficient of the highest power of $x\left(a_{n}\right)$ is greater than zero. Under the composition operation $f(x) g(x)=g(f(x)) R$ becomes a near-ring. It can be verified that $R$ is a strict fully ordered near-ring, and hence $(R,+)$ is a strict fully ordered $R$-group.

Lemma 1.7. Let $N$ be a po near-ring. A po $N$-group $G$ is a strict po $N$-group if and only if $x \in P^{*}=P \backslash\{0\}, y \in G$, and $n \in P(N)^{*}$ imply $(x+y) n-y n \in P^{*}$, where $P$ is the positive cone of the $N$-group $G$.

The proof of this lemma is easy and will be omitted.

REMARK 1.8. Let $N$ be a po near-ring. If there exists a strict po $N$-group $G$ with positive cone $P \neq\{0\}$, then $n_{1}, n_{2} \in N, n_{3} \in P(N)^{*}$ and $n_{1}<n_{2}$ imply $n_{3} n_{1}<n_{3} n_{2}$.

Proof. Let $0 \neq x \in P$. From $0<x$ and $0<n_{3}$ we have $0 n_{3}=0<x n_{3} .0<x n_{3}$ and $0<n_{2}-n_{1}$ imply $x n_{3}\left(n_{2}-n_{1}\right)=x\left(n_{3} n_{2}-n_{3} n_{1}\right)>0$. But, since $N$ is a po near-ring, we also have $n_{3} n_{1} \leqslant n_{3} n_{2}$. If $n_{3} n_{1}=n_{3} n_{2}$, then $x\left(n_{3} n_{2}-n_{3} n_{1}\right)=0$ which is a contradiction. Therefore $n_{3} n_{1}<n_{3} n_{2}$. 
Radhakrishna (1975) proved that a fully ordered near-ring $N$ with identity satisfies the condition $(-x) y=-x y$ for all $x, y \in N$. In view of this result, we are forced to impose the condition $(-x) n=-x n$ for all $x \in G$ and $n \in P(N)$ on $N$-groups $G$ to study extension to strict full orders of partial orders of strict po $N$-groups. Throughout this paper, we assume the condition $(-x) n=-x n$ for all $x \in G$ and $n \in P(N)$ on po near-rings $N$ and $N$-groups $G$.

Definition 1.9. Let $N$ be a po near-ring. Let $G$ be an $N$-group. A non-empty subset $X$ of $G$ is called a $P(N)^{*}$-cone if it satisfies the following conditions.

(i) $X$ is a normal subsemigroup of $G$; and

(ii) $x \in X, y \in G$ and $n \in P(N)^{*}$ imply $(x+y) n-y n \in X$.

LEMMA 1.10. Let $N$ be a po near-ring. Let $G$ be an $N$-group. If $X$ is a $P(N)^{*}$ cone, then $-X=\{x \in G:-x \in X\}$ is also a $P(N)^{*}$-cone.

Proof. It is clear that $-X$ is a normal subsemigroup of $G$. Let $x \in-X, y \in G$ and $n \in P(N)^{*}$. Now

$$
\begin{aligned}
-((x+y) n-y n) & =y n-(x+y) n \\
& =y n+(-(x+y)) n, \quad \text { since by our assumption }(-z) n=-z n \\
& \quad \text { for all } z \in G \text { and } n \in P(N) . \\
& =y n+(-y-x+y-y) n-(-y) n-y n \in X .
\end{aligned}
$$

Therefore $(x+y) n-y n \in-X$.

DefiNition 1.11. Let $N$ be a po near-ring. Let $G$ be an $N$-group and $S$ a subset of $G$. The intersection of all the $P(N)^{*}$-cones containing $S$ is called the $P(N)^{*}$-cone generated by $S$. We denote it by $H(S)$. We denote $H(S) \cup\{0\}$ by $H^{\prime}(S)$.

It is easy to verify the following

LEMMA 1.12. Let $N$ be a po near-ring. Let $G$ be a strict po $N$-group with positive cone $P$. Then the following hold.

(i) $a \in P$ implies $H^{\prime}(a) \subseteq P$.

(ii) $0 \neq a \in P$ implies $P \cap H(-a)=\varnothing$.

(iii) $H(-S)=-H(S)$ for all subsets $S$ of $G$.

(iv) $H^{\prime}\left(a_{1}, \ldots, a_{n}\right)=H^{\prime}\left(a_{1}\right)+\ldots+H^{\prime}\left(a_{n}\right)$ for all $a_{1}, \ldots, a_{n} \in G$.

2

In this section we obtain a necessary and sufficient condition for the extension of a strict partial order of a po $\mathrm{N}$-group to a strict full order. For this we need the following 
LeMMa 2.1. Let $N$ be a po near-ring. Let $G$ be a strict po $N$-group with positive core $P$, and let $P$ have the property:

$\left.{ }^{*}\right)$ for every finite set of nonzero elements $a_{1}, \ldots, a_{n}$ in $G$, there exists a choice of the signs $\varepsilon_{1}, \ldots, \varepsilon_{n}\left(\varepsilon_{i}=+\right.$ or -$)$ such that $P \cap H\left(\varepsilon_{1} a_{1}, \ldots, \varepsilon_{n} a_{n}\right)=\varnothing$.

Then for each nonzero element $a$ in $G$, either $P+H^{\prime}(a)$ or $P+H^{\prime}(-a)$ is the positive cone of some strict partial order of the $N$-group $G$ and has the property $\left({ }^{*}\right)$.

Proof. Let $0 \neq a \in G$. Following a procedure similar to that in po groups (Fuchs, 1963 , p. 34) we can verify that either

(i) $P^{\prime}=P+H^{\prime}(a)$ is the positive cone of some partial order of the $N$-group $G$, and $P \cap H(-a)=\varnothing$, or

(ii) $P^{\prime}=P+H^{\prime}(-a)$ is the positive cone of some partial order of the $N$-group $G$, and $P \cap H(a)=\varnothing$.

In any case, it can be verified that $P^{\prime}$ has the property $\left({ }^{*}\right)$. We show that, in either case, the partial order is strict.

Suppose (i) happens and $P^{\prime}$ is not strict. Then there exist elements $x \in P$, $y \in H^{\prime}(a), z \in G$ and $n \in P(N)^{*}$ such that $x+y \neq 0$ and $(x+y+z) n-z n=0$. Neither $x$ nor $y$ can be zero, since $P \cap H(-a)=\varnothing$ and $P$ is strict. Now we have $(x+y+z) n-(y+z) n=z n-(y+z) n \in P \cap H(-a)$ which is a contradiction. Therefore $P^{\prime}$ is strict. Similarly, if (ii) happens, we can show that $P^{\prime}=P+H^{\prime}(-a)$ is strict.

Now we are in a position to state the main theorem.

THEOREM 2.2. Let $N$ be a po near-ring. A strict partial order $P$ of a strict partially ordered $N$-group $G$ can be extended to a strict full order of the $N$-group $G$ if and only if $P$ has the property:

$\left(^{*}\right)$ for every finite set of nonzero elements $a_{1}, \ldots, a_{n}$ in $G$, there exists a choice of the signs $\varepsilon_{i}=+$ or - for $1 \leqslant i \leqslant n$ such that $P \cap H\left(\varepsilon_{1} a_{1}, \ldots, \varepsilon_{n} a_{n}\right)=\varnothing$.

Proof. Suppose that $P$ can be extended to a strict full order $P^{\prime}$. Let $a_{1}, \ldots, a_{n}$ be nonzero elements in $G$. There exist $\varepsilon_{1}, \ldots, \varepsilon_{n}\left(\varepsilon_{i}=+\right.$ or -$)$ such that

$$
\varepsilon_{i} a_{i} \in-P^{\prime *}=-P^{\prime} \backslash\{0\} .
$$

Now $-P^{* *}$ is a $P(N) *$-cone and hence $H\left(\varepsilon_{1} a_{1}, \ldots, \varepsilon_{n} a_{n}\right) \subseteq-P^{\prime *}$. Therefore we have $H\left(\varepsilon_{1} a_{1}, \ldots, \varepsilon_{n} a_{n}\right) \cap P \subseteq-P^{* *} \cap P=\varnothing$.

Conversely, suppose that $P$ has the property $\left({ }^{*}\right)$. Let $T$ be the set of all strict partial orders of $G$, which are extensions of $P$ and have the property (*). $T$ is a non-empty set since $P \in T$. It can be verified that $T$ is a partially ordered set under set inclusion and it satisfies the hypothesis of Zorn's lemma. Hence $T$ has a maximal element, say $P_{1}$. 
By Lemma 2.1, $0 \neq a \in G$ implies that either $P_{1}+H^{\prime}(a)$ or $P_{1}+H^{\prime}(-a)$ belongs to $T$. Therefore $P_{1}+H^{\prime}(a)$ or $P_{1}+H^{\prime}(-a)$ coincides with $P_{1}$. Thus $a$ or $-a$ belongs to $P_{1}$. This shows that $P_{1}$ defines a full order.

CoRollary 2.3. Let $N$ be a po near-ring. A strict partial order $P$ of a po $N$-group $G$ has no strict full extension if and only if to each $0 \neq a \in G$, there exist nonzero elements $a_{1}, \ldots, a_{n}$ in $G$ such that $P \cap H\left(a, \varepsilon_{1} a_{1}, \ldots, \varepsilon_{n} a_{n}\right) \neq \varnothing$ for every choice of the signs $\varepsilon_{1}, \ldots, \varepsilon_{n}$.

Proor. Suppose that the condition is not satisfied by $P$. Then there exists a nonzero element $a$ in $G$ such that $P \cap H\left(a, \varepsilon_{1} a_{1}, \ldots, \varepsilon_{n} a_{n}\right)=\varnothing$, for every finite set of nonzero elements $a_{1}, \ldots, a_{n}$ in $G$ for some suitable choice of the signs $\varepsilon_{1}, \ldots, \varepsilon_{n}$. This implies that $P+H^{\prime}(-a)$ is the positive cone of a strict partial order of $G$ and satisfies the property $\left(^{*}\right)$ of Theorem 2.2. So $P+H^{\prime}(-a)$ can be extended to a strict full order of $G$. This implies that $P$ can be extended to a strict full order of $G$.

Suppose that $P$ can be extended to a strict full order $P^{\prime}$ of $G$. Let $a \in G$ and $a \notin P^{\prime}$. Then $P^{\prime} \cap H\left(a, \varepsilon_{1} a_{1}, \ldots, \varepsilon_{n} a_{n}\right)=\varnothing$, for every finite set of nonzero elements $a_{1}, \ldots, a_{n}$ in $G$ for some suitable choice of the signs $\varepsilon_{1}, \ldots, \varepsilon_{n}$. This implies that $P \cap H\left(a, \varepsilon_{1} a_{1}, \ldots, \varepsilon_{n} a_{n}\right)=\varnothing$.

In this section we obtain necessary and sufficient conditions for an $\mathrm{N}$-group to admit a strict full order. We begin with the following

Definition 3.1. Let $N$ be a po near-ring. Let $G$ be an $N$-group. $G$ is called a strict orderable $\mathrm{N}$-group if it admits a strict full order.

By taking $P=\{0\}$ in Theorem 2.2, we have

THEOREM 3.2. Let $N$ be a po near-ring. An $N$-group $G$ is a strict orderable $N$-group if and only if for every finite set of nonzero elements $a_{1}, \ldots, a_{n}$ in $G$ there exists $a$ choice of the signs $\varepsilon_{1}, \ldots, \varepsilon_{n}$ such that $0 \notin H\left(\varepsilon_{1} a_{1}, \ldots, \varepsilon_{n} a_{n}\right)$.

Let $N$ be a po near-ring. Let $G$ be an $N$-group. Then the intersection of the $P(N)^{*}$-cones $H\left(\varepsilon_{1} a_{1}, \ldots, \varepsilon_{n} a_{n}\right)$, taken over all the possible choices of the signs $\varepsilon_{1}, \ldots, \varepsilon_{n}$ and fixed nonzero elements $a_{1}, \ldots, a_{n}$ in $G$, is either void or contains 0 . Therefore another characterization of Theorem 3.2 is:

THEOREM 3.3. Let $N$ be a po near-ring. An $N$-group $G$ is a strict orderable $N$-group if and only if for every finite set of nonzero elements $a_{1}, \ldots, a_{n}$ in $G$, the intersection of all the $P(N) *$-cones $H\left(\varepsilon_{1} a_{1}, \ldots, \varepsilon_{n} a_{n}\right)$ taken over all the possible choices of the signs $\varepsilon_{1}, \ldots, \varepsilon_{n}$ is void. 
The property of being a strict orderable $N$-group is thus of finite character, and consequently we have:

COROLlary 3.4. Let $N$ be a po near-ring. In order that an $N$-group $G$ be a strict orderable $N$-group it is necessary and sufficient that every finitely generated $N$-kernel of $G$ be a strict orderable $N$-group.

Proof. The proof of the necessity is obvious.

Let us assume that every finitely generated $N$-kernel of $G$ is a strict orderable $N$-group. If $G$ is not a strict orderable $N$-group, then there exist nonzero elements $a_{1}, \ldots, a_{n}$ in $G$ such that $0 \in H\left(\varepsilon_{1} a_{1}, \ldots, \varepsilon_{n} a_{n}\right)$ for every choice of the signs $\varepsilon_{1}, \ldots, \varepsilon_{n}$.

If $G_{1}$ is the $N$-kernel generated by $\left\{a_{1}, \ldots, a_{n}\right\}$, then, by our assumption, $G_{1}$ is a strict orderable $N$-group. But then $0 \notin H\left(\varepsilon_{1} a_{1}, \ldots, \varepsilon_{n} a_{n}\right)$ for some choice of the signs $\varepsilon_{1}, \ldots, \varepsilon_{n}$. Therefore $G$ is a strict orderable $N$-group.

\section{4}

Definition 4.1. Let $N$ be a po near-ring. Let $G$ be an $N$-group. $G$ is called a strict $0 *-N$-group if every strict partial order of the $N$-group $G$ can be extended to a strict full order of the $N$-group $G$.

RemarK 4.2. A strict $0^{*}-N$-group is a strict orderable $N$-group, since the trivial order of $G$ is a strict order and it can be extended to a strict full order of $G$.

THEOREM 4.3. Let $N$ be a po near-ring. An $N$-group $G$ is a strict $0^{*}-N$-group if and only if it satisfies the following conditions

(i) If $b, c \in H(a)$, then $H(b) \cap H(c) \neq \varnothing$; and

(ii) $0 \notin H(a)$ for every $0 \neq a \in G$.

Proof. Suppose that $G$ is a strict $0^{*}-N$-group. Now $G$ is also a strict orderable $N$-group, and the condition (ii) holds in any strict orderable $N$-group.

If possible, suppose that $b, c \in H(a)$ and $H(b) \cap H(c)=\varnothing$ for some $a, b$ and $c$ in $G$. It is easy to show that $P=H^{\prime}(b)+H^{\prime}(-c)$ is the positive cone of some partial order of the $N$-group $G$. We show that $P$ is strict. If $0 \neq x+y \in H^{\prime}(b)+H^{\prime}(-c)$ $\left(x \in H^{\prime}(b)\right.$ and $y \in H^{\prime}(-c)$ ) and either $x=0$ or $y=0$, then for $n \in P(N)^{*}$ and $z \in G$ we have $(x+y+z) n-z n \neq 0$ by (ii). Suppose that there exist $0 \neq x+y \in P$ $\left(0 \neq x \in H^{\prime}(b), 0 \neq y \in H^{\prime}(-c)\right), z \in G$ and $n \in P(N)^{*}$ such that $(x+y+z) n-z n=0$. This implies that $0 \neq(x+y+z) n-(y+z) n=z n-(y+z) n \in H(b) \cap H(c)$ which is a contradiction.

Therefore $P$ is a strict partial order of $G$ and hence can be extended to a strict full order $P^{\prime}$ of $G$. Now $b$ and $-c \in P$ imply that $a \in P^{\prime}$ and $-a \in P^{\prime}$ which is a contradiction. Therefore if $b, c \in H(a)$, then $H(b) \cap H(c) \neq \varnothing$. 
The proof of the converse part differs from that of the corresponding theorem in po groups (Fuchs, 1963, p. 39). In Fuchs (1963), the proof is based on the knowledge of the form of the elements in the normal subsemigroups generated by subsets of groups. But the form of the elements in $P(N)^{*}$-cones generated by subsets of $G$ is not known. Our proof makes use of Zorn's lemma. We feel that this proof is easier than that in Fuchs (1963).

Conversely, let us suppose that $G$ satisfies the conditions (i) and (ii). If $G$ is not a strict $0 *-N$-group, then there exists a strict partial order $P_{1}$ of $G$ which has no strict full extension. Let $T$ be the set of all strict partial orders of $G$ which have no strict full extension. $T$ is a non-empty set and it is a partially ordered set under set inclusion. Let $\left\{Q_{\alpha}\right\}_{\alpha \in \Delta}$ be a chain in $T$. Put $Q=\bigcup_{\alpha \in \Delta} Q_{\alpha}$. It can be verified that $Q$ is a strict full order of $G$ which has no strict full extension. Therefore $Q$ is an upper bound of the chain $\left\{Q_{\alpha}\right\}_{\alpha \in \Delta}$ in $T$. Thus by Zorn's lemma $T$ has a maximal element, say $P$. Now $P$ is not a full order. Therefore there exists $a \in G$ such that neither $a \in P$ nor $-a \in P$.

If $P \cap H(a)=\varnothing$, then $P+H^{\prime}(-a)$ defines a strict partial order of $G$ extending $P$ properly. If $P+H^{\prime}(-a)$ has a strict full extension, then it would follow that $P$ has a strict full extension. Thus $P+H^{\prime}(-a)$ belongs to $T$ which is a contradiction to the maximality of $P$. Similarly, we can show that $P \cap H(-a) \neq \varnothing$.

Let $x \in P \cap H(a)$ and $y \in P \cap H(-a)$. Now $x \neq 0$ and $y \neq 0$ by (ii). Now $x \in H(a)$ and $-y \in H(a) . \quad-y \in-P^{*}$ implies $H(-y) \subseteq-P^{*}$. $x \in P^{*}$ implies $H(x) \subseteq P^{*}$. Therefore we have $H(x) \cap H(-y) \subseteq-P^{*} \cap P^{*}=\varnothing$, which is a contradiction to (i).

Therefore $G$ is a strict $0^{*}$ - $N$-group.

The proof of the following is similar to that in po groups (Fuchs, 1963, p. 40) and shall be omitted.

COROLlary 4.4. A factor $N$-group $G / K=G^{\prime}$ of a strict $0 *-N$-group $G$ is ágain a strict $0^{*}-N$-group if and only if $G^{\prime}$ satisfies the condition (ii) of Theorem 4.3.

5

Theorem 5.1. Let $N$ be a po near-ring. A strict partial order $P$ of a po $N$-group $G$ is an intersection of strict full orders if and only if a $\notin P$ implies that for every finite set of nonzero elements $a_{1}, \ldots, a_{n}$ in $G$, there exists a choice of the signs $\varepsilon_{1}, \ldots, \varepsilon_{n}$ such that $P \cap H\left(a, \varepsilon_{1} a_{1}, \ldots, \varepsilon_{n} a_{n}\right)=\varnothing$.

Proof. Suppose that $P=\bigcap_{\alpha \in \Delta} P_{\alpha}$ where each $P_{\alpha}$ is a strict full order of $G$. Let $a \notin P$. This implies that $a \notin P_{\alpha}$ for some $\alpha \in \Delta$. Let $a_{1}, \ldots, a_{n}$ be nonzero elements in $G$. Now $a \in-P_{\alpha}^{*}$ and there exists a choice of the signs $\varepsilon_{1}, \ldots, \varepsilon_{n}$ such that $\varepsilon_{i} a_{i} \in-P_{\alpha}^{*}$ for $1 \leqslant i \leqslant n$. Thus $P \cap H\left(a, \varepsilon_{1} a_{1}, \ldots, \varepsilon_{n} a_{n}\right)$ is contained in $P \cap-P_{\alpha}^{*}=\varnothing$. 
Conversely, let $P$ satisfy the condition of the theorem. Now $a \notin P$ implies that $P^{\prime}=P+H^{\prime}(-a)$ is a strict partial order of $G$ and $P^{\prime}$ has the property $\left(^{*}\right)$ of Theorem 2.2. Therefore $P^{\prime}$ can be extended to a strict full order $P_{a}$ of $G$. But $a \notin P_{a}$ and therefore $P=\bigcap_{a_{\ddagger} P} P_{a}$.

COROLLARY 5.2. Let $N$ be a po near-ring. In a strict $0^{*}-N$-group $G$, a strict partial order $P$ of $G$ is an intersection of strict full orders of $G$ if and only if $a \notin P$ implies that $P \cap H(a)=\varnothing$.

Proof. If $P$ is an intersection of strict full orders, then the condition holds by Theorem 5.1. If the condition holds, then $a \notin P$ implies that $P+H^{\prime}(-a)$ is a strict partial order of $G$ which can be extended to a strict full order $P_{a}$ of $G$, and $a \notin P_{a}$. Therefore $P=\bigcap_{a \notin P} P_{a}$.

\section{Acknowledgement}

I take this opportunity to thank my research director Professor D. Ramakotaiah for suggesting this problem and for his valuable guidance.

\section{REFERENCES}

L. Fuchs (1963), Partially Ordered Algebraic Systems (Pergamon Press, Addison-Wesley). G. Pilz (1971), "Direct sums of ordered near-rings", J. Algebra 18, 340-342.

A. Radhakrishna (1975), "On lattice ordered near-ring and nonassociative rings" (thesis submitted to the Department of Mathematics, Indian Institute of Technology, Kanpur, India).

Department of Mathematics

Nagarjuna University

A.C. College Campus

Guntur, A.P. (India) 